\title{
La question du pouvoir exécutif dans l'évolution institutionnelle et le débat politique révolutionnaire
}

Paolo Colombo

\section{(2) OpenEdition \\ Journals}

Édition électronique

URL : https://journals.openedition.org/ahrf/98

DOI : $10.4000 /$ ahrf.98

ISSN : 1952-403X

Éditeur :

Armand Colin, Société des études robespierristes

Édition imprimée

Date de publication : 1 mars 2000

Pagination : 1-26

ISSN : 0003-4436

\section{Référence électronique}

Paolo Colombo, « La question du pouvoir exécutif dans l'évolution institutionnelle et le débat politique révolutionnaire », Annales historiques de la Révolution française [En ligne], 319 | janvier-mars 2000, mis en ligne le 11 mai 2006, consulté le 22 avril 2022. URL : http://journals.openedition.org/ahrf/98 ; DOI : https://doi.org/10.4000/ahrf.98

Ce document a été généré automatiquement le 22 avril 2022.

Tous droits réservés 


\title{
La question du pouvoir exécutif dans l'évolution institutionnelle et le débat politique révolutionnaire
}

\author{
Paolo Colombo
}

Une introduction à la théorie constitutionnelle de la Révolution : légicentrisme vrai ou présumé?

1 On ne peut pas nier que le débat politique sous la Révolution apparaît essentiellement centré sur la question législative. Et il est également évident que, assez souvent, les interventions des députés révolutionnaires semblent par conséquent reléguer en position marginale le problème du pouvoir exécutif ou, au moins, montrent une sorte d'hostilité envers ce pouvoir. «Toutes les constitutions françaises sont des batteries dirigées contre le pouvoir exécutif ", écrit même Édouard Laboulaye ${ }^{1}$. Mais, bien que le système projeté après 1789 puisse être considéré en effet comme légicentriste (ou "législatif-centriste " $)^{2}$, il faut reconnaître que la théorie constitutionnelle et institutionnelle de la Révolution analyse avec continuité et profondeur la question du pouvoir exécutif ${ }^{3}$. Ce facteur est trop souvent oublié dans les reconstructions de l'évolution théorique révolutionnaire, et il n'est pas le seul.

2 Si l'on accepte que l'effort des "architectes politiques $»^{4}$ de la Révolution se borne à l'instauration d'un régime fondé sur la production de lois et si l'on croit pouvoir réduire le problème du gouvernement à la lutte contre la monarchie, on court le risque de perdre de vue la grande complexité de la réflexion théorique des dix années révolutionnaires.

3 Dix ans : il faut le souligner immédiatement. Les divisions qui ont permis de partager en périodes la Révolution ont certes leur justification et on ne veut pas retomber ici dans le débat sur la continuité et les fractures révolutionnaires. Chaque moment (la monarchie constitutionnelle comme la Terreur, le Directoire comme le Consulat) révèle ses particularités politiques et chaque partage historique a son utilité heuristique. Cependant, ce qu'on veut rappeler, c'est que les « lignes théoriques » fondamentales de la pensée révolutionnaire sont souvent fortes, précises et continues au-delà de ces 
mêmes partages. Compte tenu de la nécessité de résoudre les problèmes contingents ou du facteur d'homogénéité constitué par la permanence au cœur des événements politiques de personnages capables de traverser indemnes les phases les plus dramatiques du procès de régénération de l'Ancien Régime, la Révolution montre de ce point de vue des continuités précises. Il existe des "paradigmes théoriques ${ }^{5}$ qui orientent le débat ainsi que la pratique révolutionnaire. C'est exactement de ces " filons» théoriques que les constitutionnalistes et les hommes politiques des xIxe et xxe siècles tireront en grande partie les matériaux nécessaires à leurs constructions politiques. À ce titre, il faut noter que la conception française de chef d'État, qui reste par ailleurs peu étudiée, représente probablement dans ce sens l'un des cas les plus emblématiques ${ }^{6}$.

4 La pensée constitutionnelle révolutionnaire, de plus, est rigoureusement " circulaire ", c'est-à-dire que chaque élément qui la compose se soude à un ou plusieurs autres éléments dans une chaîne logique qu'on voudrait impossible à briser. Et cela implique, naturellement, une grande œuvre de redéfinition en même temps conceptuelle et linguistique ${ }^{7}$. Pas un seul vide ne peut être accepté dans la nouvelle construction politique élevée par la Révolution. "Combien de choses à observer dans la consolidation d'un nouvel établissement public!»- écrira le feuillant Pierre Lacretelle - «Pas un obstacle ne doit être laissé dans l'espace qu'il doit remplir; pas une ouverture n'y doit rester à l'invasion d'un esprit contraire $»^{8}$. Un véritable horror vacui tenaille les Constituants révolutionnaires. Les débats parlementaires montrent clairement que chaque problème politique est formulé - presque nécessairement - en contact avec toutes les questions constitutionnelles apparentées. Le nouveau système devrait "tenir " lorsqu'il serait soumis à l'épreuve abstraite de la critique rationnelle ou à la vérification pratique de la vie institutionnelle. Il ne faut pas oublier cette prémisse: cela nous permet de comprendre pourquoi tous les détails deviennent essentiels pour les députés constituants de la Révolution ${ }^{9}$ et pourquoi ces derniers s'engagent obstinément à inclure chaque composant de la nouvelle formule politique dans le «cercle $»^{10}$ de la Constitution écrite (qui représente la manifestation la plus concrète de leur théorie).

5 Le pouvoir exécutif ne fait pas exception: on le voit aisément en ce qui concerne la question de la royauté, que les législateurs des années $1789-1791^{11}$ essaient sans succès de concilier harmonieusement avec les autres éléments constitutionnels. Ce n'est pas un hasard si la première apparition officielle de l'expression "monarchie constitutionnelle » semble pouvoir être datée de $1791^{12}$. Rendre «constitutionnelle » la monarchie revient toutefois à s'interroger sur la signification précise de la représentation politique (le roi, est-il représentant ou pas ? Représenter, cela signifiet-il « vouloir » ou " agir » ? quelle liaison existe-t-il entre représentation et élection ?), sur les fonctions législatives à reconnaître à Louis XVI (le veto est-il activité législative ?) et éventuellement sur son rôle constituant (si le Roi est législateur, pourrait-on nier qu'il soit aussi constituant?), sur les limites des pouvoirs constitués (que signifie exactement exécuter ?) et donc sur la valeur de la Constitution (est-elle l'acte de partage des pouvoirs?). "Tout est lié, tout est enchainé », écrira Antoine Barnave ${ }^{13}$.

6 Justement le débat sur le veto est, en ce sens, emblématique. C'est vrai que septembre 1789 nous montre un important combat politique sur le sort futur de la royauté, mais cette lutte nous cache très souvent qu'on se dispute déjà sur la validité de la fracture 
que la Révolution est en train de réaliser, entre "volonté " et "action». Le gouvernement ne peut pas coïncider avec l'Exécutif : «le Pouvoir Exécutif est la force motrice d'un Gouvernement; il représente, dans un système politique, cette puissance mystérieuse qui, dans l'homme moral, réunit l'action à la volonté » - avait déjà mis en garde Jacques Necker ${ }^{14}$.

On ne peut pas trop s'arrêter ici sur les complexes interactions entre "constitution", " gouvernement » et " exécutif », mais il est possible de saisir intuitivement que l'idée de pouvoir exécutif est l'instrument qui permet d'intégrer le gouvernement dans la Constitution. Volonté d'un côté et action de l'autre: une solution différente - dira toujours Antoine Barnave - signifie « prendre une forme moins constitutionnelle $»^{15}$. De cette façon, deux vigoureuses tendances (l'une plus visible, l'autre moins évidente, mais pas moins forte) de la théorie révolutionnaire entrent en conflit : d'une part, on projette un système qui sépare le moment de la décision de celui de l'exécution, de l'autre on essaie de maintenir en vie, ou de ressusciter, l'union de "vouloir » et d'« agir»"

8 Apparemment, le schéma constitutionnel de la division des pouvoirs, surtout celui du début de la Révolution, marque fortement la fracture entre les deux pouvoirs en question. Et l'affirmation par laquelle le Roi gouverne «suivant la loi » ${ }^{16}$, n'est qu'une recomposition postiche de cette fracture. On se retrouve de toute façon aux prises avec le même problème, qui - pour Henry Bancal - «est donc de trouver une forme de pouvoir exécutif, où la volonté de celui qui exécute puisse être toujours conforme à celle de la loi ${ }^{17}$. Quand les députés révolutionnaires débattent du veto royal et du droit de guerre et de paix, au fond, ils discutent de comment éliminer la contradiction institutionnelle entre volonté et action, peut-être plus encore que des destinées de la monarchie.

9 Les membres de l'Assemblée disent plusieurs fois et très clairement que les attributions du monarque doivent être considérées dans une logique strictement constitutionnelle : "C'est sous le point de vue des principes de la Constitution que cette cause doit être discutée ", déclare Gaultier de Biauzat en parlant du droit de guerre et de paix ${ }^{18}$. Et la Constitution est essentiellement «acte de partage des pouvoirs ${ }^{19}$. Les contenus institutionnels des discussions sur le veto sont donc avant tout incarnés par le problème de l'intégration entre pouvoir législatif et pouvoir exécutif et de la définition de leurs limites d'action. Dans ce sens, on peut dire que l'urgence historique de la question monarchique a voilé de plusieurs points de vue les aspects les plus profonds d'un débat centré en premier lieu sur l'Exécutif (qui est en soi quelque chose de différent du roi).

D'autre part, l'attention des études sur ce moment de la vie parlementaire révolutionnaire a été attirée le plus souvent par la question représentative, mais encore une fois, si l'on est attentif, au-dessous d'elle demeure le binôme vouloir/agir, qui se trouve même pour la première fois posé avec une telle décision sur la table du jeu constituant.

11 Il arrive justement qu'un point central du débat sur le veto, où se suivent à un rythme soutenu les opinions de Roederer, Robespierre, Thouret et Barnave, se déroule autour de ce binôme là. Roederer explique que la représentation n'est pas obligatoirement liée à l'élection: on peut être représentant pour «agir » mais non pour "vouloir », et inversement. De ce point de vue, même les administrateurs, dépositaires d'un simple « pouvoir commis », pourraient se considérer « représentants $»^{20}$. 

«fonctions » (qui au contraire peuvent être déléguées). De cette distinction dérivent plusieurs conséquences, développées aussi par Rabaut Saint-Étienne. Le Législatif, puisqu'il est expression de volonté, réside dans le peuple/nation qui a toujours le droit de l'exercer (pouvoir constituant), tandis que l'Exécutif, qui est essentiellement action, «appartient souverainement» à la nation même mais doit nécessairement être délégué ; et cette délégation (au roi), selon Robespierre qui reprend à ce propos des idées de Roederer, ne produit pas de représentation car elle ne vient pas du choix électif ${ }^{21}$. qu'Emmanuel Sieyès aurait dû tenir un discours où il aurait répliqué que la question était ainsi mal posée parce que chaque fonction publique implique l'exercice d'une forme de volontées.

Nous pouvons passer sur les motifs secrets, sur les objectifs finaux, sur les succès stratégiques, vrais ou présumés, obtenus par les orateurs que nous venons de citer, car ce qui nous intéresse ici ce sont les catégories utilisées (vouloir/agir, volonté/action), qui se trouvent pour ainsi dire en amont de la question même de la représentation. Cela nous indique qu'une bonne partie des énergies des ingénieurs constitutionnels de la Révolution est consacrée à l'étude de solutions pour le problème exécutif déjà à partir de la période monarchique.

Bien sûr: l'Exécutif se lie, au début de la Révolution, avec la royauté et dans la Constitution de 1791 l'idée de gouvernement comme simple exécution l'emporte. Mais les Monarchiens (Stanislas de Clermont-Tonnerre en tête) esquissent déjà l'image d'un roi «pouvoir neutre $»^{24}$; Mirabeau aspire à un régime parlementaire ${ }^{25}$ qui sépare les pouvoirs mais oblige à réunir les fonctions; Sieyès repère une volonté dans toute fonction publique; parmi les députés il y en a qui proposent un chef (royal, naturellement) de l'Exécutif comme "centre de tous les mouvements du corps politique " et qui rêvent d'un chef d'État électif ${ }^{26}$; les discussions parlementaires mettent souvent en garde à propos des risques de paralysie institutionnelle venant d'une séparation trop rigide des pouvoirs. Bref, à côté de la grande lutte pour ou contre la monarchie, la Révolution commence très tôt à réfléchir sur l'essence constitutionnelle de la question du gouvernement.

L'héritage caché : l'efficacité du gouvernement des Comités

17 Les positions de l'historiographie à propos de la période du gouvernement des Comités sont souvent assez semblables à celles qui concernent la monarchie constitutionnelle. Interprétées traditionnellement comme expérience exceptionnelle (la Constitution dort dans l'arche de cèdre aux pieds du président de la Convention jusqu'à la paix), les deux années qui vont de 1792 à 1794 sont en effet observées à travers le filtre du pragmatisme : les problèmes de la guerre, le tribunal révolutionnaire, la dictature d'un comité de l'Assemblée, le renversement du mécanisme représentatif, le recours à la démocratie directe, les insurrections populaires, la ferveur des sections... sont les 
éléments avec lesquels on décrit l'intervalle entre monarchie et Directoire. De ce point de vue, la Terreur n'est guère plus que la Terreur.

Mais on ne peut pas croire que la Convention (et donc ses deux comités) se place au centre du système politique en dehors de tout esprit doctrinaire. Déjà les débats des années 1789-1791, en traitant surtout du Conseil des ministres, avaient abordé le terrain de l'Exécutif collégial ${ }^{27}$ : idée reprise par Condorcet dans son plan constitutionnel de février 1793 et discutée dans le débat suivant. "Unité d'action ", " surveillance ", « donner du nerf à l'exécutif » commencent à devenir des mots clés de l'élaboration théorique révolutionnaire. Commence aussi en même temps à s'imposer en préparation à l'idée de "gouvernement révolutionnaire" - la notion de "gouvernement représentatif » et ses implications ne doivent pas être sous-estimées. La signification de "gouverner", aussi bien que celle de "représenter " doivent en effet être vérifiées ${ }^{28}$. Le passage qui amène à appliquer la qualification de représentant (typique jusque-là des assemblées législatives) à l'Exécutif n'est pas simple, et, d'autre part, les Comités de salut public et de sûreté générale répondent dans la pratique à une exigence d'efficacité dans le gouvernement qui se manifeste désormais de plusieurs façons.

Les projets envoyés à la Convention depuis la présentation du plan girondin de Constitution témoignent d'une ardente activité intellectuelle sur ces points. Il s'agit, en effet, de perfectionner la démocratie indirecte: Didier Thirion, par exemple, précise que dans un gouvernement représentatif, «toutes les autorités constitituées y sont temporaires et électives", alors que pour Pierre Charles François Dupont «le gouvernement français est une délégation représentative $»^{29}$. Mais il faut penser en même temps à un Exécutif qui ne soit plus monarchique. Il y a donc deux phases d'expérimentation institutionnelle : celle du Conseil exécutif provisoire de l'été 1792 et celle du Comité de défense générale de mars 1793 que Paul Bastid a interprété de manière significative comme « une sorte de grand ministère d'union nationale » et qui se trouve à l'origine de la naissance du Comité de salut public ${ }^{30}$. Les problèmes en jeu sur ces terrains sont fondamentaux.

Il n'est pas fortuit que deux personnages comme Boissy d'Anglas et Daunou prennent position directement sur ce difficile champ de bataille. Bien qu'on soit en train de préparer la parthénogenèse institutionnelle à travers laquelle la Convention produira le "gouvernement révolutionnaire" dans son sein, les deux hommes politiques demeurent sur le terrain de la séparation des pouvoirs et incarnent ainsi une ligne théorique dirigée vers l'élaboration d'un Exécutif collégial et électif : une ligne qui se prolonge jusqu'à la doctrine constitutionnelle directoriale.

21 Boissy d'Anglas ( J'ai rétabli le corps électoral pour la nomination des agents du pouvoir exécutif ») propose un Conseil exécutif national composé de quinze membres choisis par des électeurs nommés par le peuple et présidé alternativement par l'un d'eux ; l'autorité de ce Conseil est conçue comme indivisible, le titre de ministre devant être aboli ainsi que les divisions ministérielles.

Daunou, au contraire ( Je ne conseillerai pas [...] de faire choisir les ministres par l'universalité des citoyens »), pense à un Conseil de vingt-cinq ministres nommés par le Législatif et divisé en cinq sections (justice, force publique, finances publiques, administration intérieure, affaires étrangères $)^{31}$.

Certes, on ne doit pas oublier que même Condorcet, bien qu'attribuant une suprême importance au pouvoir législatif, propose un Exécutif collégial dont les membres 
seraient élus cette fois directement par le peuple. Le débat sur ces points, lors des six premiers mois de 1793, est plus profond qu'on le croit ${ }^{32}$.

On sait que le discours jacobin renverse l'idée traditionnelle de la représentation ${ }^{33}$ et, avec elle, le problème du gouvernement «représentatif»: "gouvernement » et "représentation » sont liés de façon à placer au premier plan le rapport entre pouvoir politique et base sociale. Ce rapport n'est pas observé à la lumière de la légitimation électorale mais plutôt, dans la direction opposée, à celle de la correspondance entre représentants et représentés.

Or, sans rentrer dans le problème d'évaluer la période comprise entre la fin de 1792 et le 9 thermidor comme expérience de gouvernement parlementaire (hypothèse déjà suggérée par Alphonse Aulard et, dans son sillage, par Boris Mirkine-Guetzevitch) et sans aller jusqu'à juger les aspects antilibéraux, autoritaires et dictatoriaux de la Terreur, il faut relever que des points fondamentaux en matière de référence théorique sont mis à jour, points auxquels on se rapportera dans les années suivantes.

Il faut «donner du nerf au gouvernement » et assurer à la République, dit BillaudVarenne ${ }^{34}$. Il nous semble ainsi voir renaître le problème du rapport entre « vouloir » et " agir ", mais la perspective est différente par rapport aux années 1789-1791. La racine $\mathrm{du}$ problème se trouve maintenant dans le pouvoir de gouverner. On sort de l'antinomie pensée/action, ou représentation/décision, parce qu'on recommence à concevoir un gouvernement comme somme des deux éléments.

C'est pourquoi, en déplaçant l'analyse directement de la Terreur au Directoire, on perd un enchaînement théorique fondamental : celui qui, justement, montre la récupération de l'expérience du gouvernement révolutionnaire.

On néglige en effet assez souvent deux moments du débat parlementaire qui marquent le passage du régime jacobin au système thermidorien : celui sur les lois organiques pour l'éventuelle application de la Constitution de 1793 (novembre 1794-mars 1795) et celui sur le gouvernement provisoire (printemps 1795). Il est évident qu'à l'origine de l'essai de récupérer l'acte constitutionnel de l'an I il y a peu de volonté politique réelle, sous peine de légitimer une dangereuse conception «maximaliste » de la souveraineté populaire ${ }^{35}$, et beaucoup d'esprit stratégique : au moins, les thermidoriens ne peuvent pas proposer tout d'un coup l'abolition (illégale, d'où qu'on la regarde) de la Constitution la plus démocratique qu'on n'ait jamais produite. Mais, en même temps, on ne peut point fermer aujourd'hui les yeux sur l'importance des sujets traités à ce moment là, ni croire qu'il s'agit simplement de discussions en réaction à la Terreur visant à réduire la force de l'Exécutif. Il suffit de penser que, déjà dans les prodromes de ces débats, à la fin de l'été 1794, Chasles pose « une grande question à examiner : c'est celle de savoir si, sans inconvénients pour le gouvernement révolutionnaire, le peuple ne peut pas être appelé à choisir les individus qui doivent composer le comité... ${ }^{36}$. Le point est essentiel. Faudrait-il projeter un Exécutif électif (en acceptant toutes les conséquences qui en dérivent, par exemple sur le plan représentatif)?

Avant tout, évidemment, il faut préciser la signification exacte de l'expression "gouvernement révolutionnaire ", qui ne peut plus correspondre à celle acceptée par les Montagnards. Dans ce sens, les séances de l'hiver 1794 préparent le débat du printemps sur le gouvernement provisoire, qui à son tour montre à l'avance presque tous les éléments des discussions constituantes suivantes. Donc, un gouvernement est-il « révolutionnaire " quand il est " propre à achever la Révolution, ou bien en agissant à la manière de la Révolution ?»? - demande réthoriquement Tallien, pour répondre 
immédiatement qu'il est celui qui «seul peut et doit nécessairement achever et assurer la Révolution ". Encore une fois, tout se tient dans la construction théorique de la révolution. Le gouvernement révolutionnaire devient «le complément de l'ordre essentiel de la société politique ». Il faut terminer la Révolution et assurer l'ordre (ce qui signifie, dans le langage constituant, affirmer avant tout une Constitution). Et la question se réduit ainsi, emblématiquement, à repérer "les conditions qu'il [le gouvernement] doit remplir pour parvenir à cette fin ». Ce n'est pas un hasard si, à partir de là, on arrive à demander les lois organiques pour l'établissement de la Constitution et à transformer le gouvernement « révolutionnaire » en gouvernement « républicain »:

« Nous devons conduire le char de la Révolution jusqu'au but de la carrière qu'il a à parcourir ; mais nous devons, pendant sa course, préparer les lois conservatrices de l'acte constitutionnel, afin qu'il n'y ait aucun intervalle entre l'anéantissement du gouvernement révolutionnaire et l'établissement du gouvernement républicain ", déclare Audouin ${ }^{37}$.

Parallèlement au débat sur les lois organiques se déroule celui sur le gouvernement provisoire, ce qui atteste la forte liaison entre Constitution, établissement de l'ordre, transition de régime et gouvernement.

Unité d'action et Exécutif collégial : le débat sur le Directoire

La Constitution de l'an III - comme il arrive souvent dans ces cas - n'est pas uniquement le fruit des discussions strictement constituantes. Beaucoup de ses racines se trouvent ailleurs et en particulier justement dans le débat sur le gouvernement provisoire. Les problèmes abordés pendant la recherche de solutions pour la réorganisation des Comités montrent que l'acte constitutionnel thermidorien est fils de la question gouvernementale: ses caractères génétiques sont déjà inscrits dans les réflexions rétrospectives sur le gouvernement révolutionnaire. Tant il est vrai que cet acte donne la vie à un régime qui prend son nom de l'organe exécutif.

Immédiatement après la chute de Robespierre, il apparaît déjà évident qu'il faut un Exécutif fort : «le pouvoir exécutif est l'axe sur lequel tourne la machine politique »soutient Cambacérès ${ }^{38}$. Et, d'autre part, le point le plus intéressant de ce débat est probablement celui où Thibaudeau s'engage à démontrer, à la tribune mais aussi dans ses écrits, que "l'ancien gouvernement était bon, à l'exception de la tyrannie qu'il exerçait». Sur ce sujet interviennent aussi, parmi d'autres, Lanjuinais, Louvet, Villetard, Fréron, Cambacérès ${ }^{39}$. Nous pouvons observer ici à l'avance les éléments d'une confrontation qui divisera de façon évidente le front libéral quelques années après : d'un côté les défenseurs du pragmatisme jacobin de gouvernement, dramatique mais indispensable point de passage vers la république, et de l'autre ses détracteurs, guère disposés à troquer la liberté politique pour la liberté civile. Il s'agit d'une confrontation qui engendrera en premier lieu surtout la polémique entre Adrien LezayMarnesia et Benjamin Constant ${ }^{40}$ et qui sera destinée à continuer longtemps.

Au-delà du difficile jugement sur la Terreur, ce que les discussions soulevées par Thibaudeau montrent clairement est la consolidation d'une idée : «le pouvoir exécutif consiste dans la pensée du gouvernement ». Il ajoute que, depuis cinq ans, on pense à la France comme marchant "pour ainsi dire, sans système en politique $»^{41}$.

Le thème de la pensée du gouvernement, repris avec force et perfectionné par Barère ${ }^{42}$, s'insère dans le cadre plus général de la recherche d'un nouveau système, qui est avant tout un nouveau système de division et d'organisation des pouvoirs. Il faut combattre 
en même temps la "dissémination" et la "concentration absolue » des pouvoirs. "L'unité dans le gouvernement est l'unité d'action, et non la réunion de tous les pouvoirs ", dit Cambacérès. Et, plus d'un an après, Lenoir-Laroche rappelle encore que « la séparation des pouvoirs n'est pas l'opposition des pouvoirs. Ils doivent être séparés pour garantir la liberté; ils doivent agir de concert pour qu'il y ait un gouvernement $»^{43}$.

Comment assurer alors le juste équilibre de la machine politique? Si l'on va chercher les coordonnées de l'élaboration théorique thermidorienne seulement dans le résultat représenté par la Constitution de l'an III, on n'arrive pas à en percevoir les vraies dimensions : Mme de Staël remarquait déjà dans une lettre à Pierre-Louis Roederer que " on parle beaucoup de la division des pouvoirs; et c'est peut-être leur union le problème le plus difficile $»^{44}$. Seulement de ce point de vue - comme le montre bien Mme de Staël en déplaçant, immédiatement après, son analyse sur le terrain de l'Exécutif et en particulier sur celui du choix des ministres dans le Législatif -, on peut comprendre l'effort des ingénieurs constitutionnels à propos de la structure du gouvernement.

Il suffirait, à ce propos, de consulter les papiers de la Commission des Onze. Les notes manuscrites des travaux montrent que l'élaboration constitutionnelle ne fut pas univoque et que deux ordres de solutions furent pris en considération: d'un côté les raisons en faveur d'un magistrat exécutif unique, électif et temporaire, de l'autre celles pour un corps exécutif collégial ${ }^{45}$. Ce schéma de travail est aussi confirmé, indirectement, par Pierre-Louis Roederer, "douzième " membre de la Commission ${ }^{46}$. Dans le premier sens, des éléments importants entraient en jeu : la plus grande facilité de limitation d'un pouvoir plus concentré, le risque que la division de l'Exécutif se traduise dans une tyrannie du plus fort ou dans une guerre continuelle, la peur de se trouver en face de nombreux "Grands Citoyens ", la nécessité d'éviter - à travers la centralisation - un glissement du gouvernement vers l'aristocratie. Mais l'argument le plus intéressant concerne la façon dont un magistrat unique permet d'allier l'inviolabilité avec la responsabilité : on reproduit de cette façon un élément essentiel du schéma classique de la monarchie constitutionnelle. Dans l'autre sens, et ce n'est pas un hasard, la crainte que «malgré toutes les précautions... [un agent unique] retiendra toujours quelque simulacre des formes royales " est immédiatement évoquée. De plus, dans un corps collégial, les délibérations seront plus réfléchies et l'exécution - confiée à un seul membre - sera rapide et prompte. La présence d'un chef unique, enfin, implique celle d'un Conseil et l'expérience des régimes monarchiques prouve qu'en général ce dernier finit par gouverner.

37 L'hypothèse d'un chef exécutif monocratique mais pas monarchique est donc prise sérieusement en considération. Les papiers de la Commission montrent que les constituants évaluent l'idée d'un «agent unique » (avec autant de ministres adjoints nommés par lui-même et correspondant aux branches de l'administration), doué de veto suspensif, élu par des électeurs envoyés de chaque district et choisis dans une liste présentée par le Corps législatif. Même un mécanisme de confiance de l'Assemblée est mis à l'étude, pour une utilisation tantôt envers le chef unique, tantôt envers chacun de ses ministres $^{47}$; ce point est évidemment très important dans la perspective de l'évolution parlementariste du régime constitutionnel.

D'autre part, les sources d'archives nous révèlent que l'idée d'élire un vrai président s'est désormais frayée un premier chemin dans le débat politique : un projet anonyme 
parvenu à la Commission ${ }^{48}$ nous le signale, pour ainsi dire en négatif, quand on $\mathrm{y}$ propose que - en supposant que les Onze ne veuillent pas accepter l'élection d'un président - l'Exécutif soit délégué à deux consuls (l'un pour l'extérieur, l'autre pour l'intérieur) et à un Conseil de six ministres. Donc, d'une certaine façon, le régime présidentiel commençait déjà à se dessiner. Mais il faut tout de même reconnaître que ce débat se déroule dans plusieurs directions différentes : jusqu'à l'attribution, par exemple, d'une sorte de pouvoir exécutif à une deuxième chambre ${ }^{49}$. Dans un tableau tellement articulé, la réflexion constituante de l'an III paraît avoir précisé du moins une importante conviction: le gouvernement n'est pas de la simple exécution. Il faut distinguer "volonté » et " action ", " vouloir » et " agir » du gouvernement même (ou justement, selon les mots de Thibaudeau, "gouvernement» et "exécution») et le centre de la pensée exécutive se trouve au même niveau que celui du Législatif: «le pouvoir législatif est la volonté qui médite, mûrit et crée la loi » - affirme François Poultier - «la puissance exécutive doit être la volonté prompte et libre qui l'exécute $\aleph^{50}$.

39 Le débat parlementaire est lui aussi assez vif bien que le refus enfin opposé par la Commission à la possibilité d'un chef unique et à l'élection directe de l'Exécutif soit clairement manifesté par Boissy d'Anglas au moment de présenter le projet à l'Assemblée ${ }^{51}$; toutes les questions liées à la construction du gouvernement sont de nouveau mises en jeu dans les séances de l'Assemblée : caractère représentatif de l'Exécutif, élection directe et indirecte, composition monocratique ou collégiale, rapport, équilibre et solution des conflits entre les pouvoirs ${ }^{52}$.

40 Et le moment où la discussion révèle les plus intimes connections entre Exécutif, Législatif et pouvoir «neutre » s'avère peut-être l'un des plus intéressants parce que le jury constitutionnaire proposé par Sieyès est ici mis en question. Le 30 thermidor JeanFrançois Ehrmann, député apparemment de second plan dans les jeux politiques de l'Assemblée, prend la parole pour demander pourquoi, si « la Convention nationale a rejeté l'idée ingénieuse de notre collègue Sieyès sur le jury constitutionnaire [...] », on ne cesse pas « de réclamer de tous côtés contre la faiblesse du pouvoir exécutif » ${ }^{53}$. Et il continue : "Je ne puis m'empêcher de croire qu'il y ait une lacune, quelque part que ce soit, dans les liaisons du pouvoir exécutif avec les autres pouvoirs ». Ehrmann s'oppose expressément à un Directoire simple exécuteur, soulève le problème des relations entre pouvoirs constitués et finit par demander un veto suspensif pour le gouvernement. Cette proposition ne passera pas, nous le savons. Mais elle n'est pas pour cela moins importante.

Le discours d'Ehrmann, en effet, n'est point improvisé, ni occasionnel. Ses mots ne sont rien d'autre que l'illustration d'un courant de pensée qui ne croit pas à la possibilité de réaliser un système de complète et parfaite séparation des pouvoirs. La sollicitude des interventions de Daunou et Lanjuinais ${ }^{54}$ en faveur d'Ehrmann permet de les ranger parmi les membres de ce courant (et nous révèle une stratégie qui consiste à tester les réactions par l'intermédiaire d'un député plus « obscur »). Mais derrière la proposition d'Ehrmann, il y aurait aussi Mme de Staël et - ce qui n'est pas improbable - l'entière Commission des Onze ${ }^{55}$. Le groupe de théoriciens favorables à une division moins rigide des pouvoirs était donc bien influent !

42 La liaison établie par Ehrmann entre le projet de Sieyès (sur lequel on va revenir) et le rôle du gouvernement dans le nouvel édifice constitutionnel prouve ainsi que le problème essentiel, en l'an III, est encore celui d'assurer l'équilibre des pouvoirs: le 
problème de la "pierre philosophale en politique ", dit Thibaudeau ${ }^{56}$. Il y a plusieurs solutions imaginées: équilibre automatique dépendant des dispositions constitutionnelles, mécanismes de responsabilité du gouvernement et des Chambres du type à peu près parlementaire, pouvoir modérateur (ou neutre) chargé de rétablir la balance institutionnelle en cas de besoin. Mais si l'on regarde seulement dans les articles de la Constitution, naturellement, on n'y voit que la première de ces solutions. Solution, d'autre part, absolument cohérente avec la logique révolutionnaire: l'équilibre parfait trouve sa raison d'être dans la Constitution. Ou, comme Michel Troper l'a justement souligné, la Constitution est si bien organisée qu'il est impossible de la violer ${ }^{57}$. On ne veut pas « interdire » mais « empêcher ».

La deuxième solution - qui cherche l'équilibre constitutionnel dans la dynamique politique et qui sera soutenue significativement par Benjamin Constant dans la forme du veto à l'Exécutif et dans le droit de dissolution des Chambres quand gouvernement et Législatif se trouvent en conflit ${ }^{58}$-- entre en jeu (comme le montre l'intervention d'Ehrmann) si on refuse la troisième : l'équilibre dans la dynamique institutionnelle, c'est-à-dire le pouvoir neutre défendu avant tout par Emmanuel Sieyès.

Le discours tenu par l'abbé le 2 thermidor, bien qu'exceptionnel dans le panorama parlementaire de la Révolution, ne représente pas un merle blanc, comme l'on a cru pendant longtemps. Au contraire il est emblématique d'une ligne de pensée assez répandue : Roederer et Daunou - qui sera rédacteur de la Constitution - en sont aussi partisans et on peut en trouver des traces jusque dans les papiers de la Commission des Onze ${ }^{59}$.

Sans qu'il soit possible de s'attarder sur le complexe projet de Sieyès ${ }^{60}$, dont les contenus sont désormais assez connus, il suffit pour l'instant de noter les trois points suivants. Premièrement, Sieyès est le porte-étendard de ceux qui pensent qu'il faut " compliquer les pouvoirs $»^{61}$ : ce qui nous révèle une image bien plus articulée de la pensée constituante révolutionnaire par rapport à celle fondée sur la tripartition de Montesquieu ou, mieux, sur la simple dynamique des pouvoirs législatif et exécutif. Deuxièmement, la création d'un pouvoir neutre et l'idée de compliquer les pouvoirs sont strictement liées à une nouvelle conception du gouvernement centrée avant tout, comme le montrent les mots de l'abbé et l'intervention d'Ehrmann, sur la conviction qu'il doit unir "pensée » et «action ». Ce déplacement de l'Exécutif sur le même plan que le Législatif est pour ainsi dire contrebalancé par la faculté donnée à un pouvoir modérateur de résoudre les conflits entre Chambres et Gouvernement. Tout ce système ne peut être enfin compris sans renvoyer à la distinction fondamentale entre pouvoir constituant et pouvoirs constitués : l'essence même du pouvoir neutre a affaire aux plus hauts niveaux de la décision politique et de la production des lois (il suffit de penser à son rôle d'arbitre entre les pouvoirs constitués ou à sa fonction dans la révision de la Constitution). Encore une fois, tout est enchaîné dans la théorie constitutionnelle de la Révolution.

Mais, encore une fois, cette chaîne théorique risque de se trouver enfin à un point mort : «quis custodiet ipsos custodes ? $»^{62}$. C'est-à-dire que, selon les mots de Thibaudeau, si « on donne aussi des surveillants à ce jury [...] cette surveillance graduelle s'étendrait à l'infini $»^{63}$. Mais on ne doit pas juger uniquement de ce point de vue les constituants de l'an III : ni l'imparfaite tenue logique de ses constructions, ni le refus parlementaire opposé à quelques-unes d'entre elles ne les condamnent. Au contraire, le débat luimême sur le pouvoir neutre nous montre que, paradoxalement, quand la théorie 
constitutionnelle de la Révolution s'applique plus encore qu'auparavant à réaliser un système politique complètement réglé par les articles de la Constitution, ses auteurs comprennent que cet effort est inutile.

«Je pense toujours que rien n'est arbitraire dans la mécanique sociale, et que la place de chaque pièce se trouve déterminée par des rapports qui ne dépendent pas de la simple volonté du mécanicien ", affirme Sieyès ${ }^{64}$. La machine politique a ses propres règles (ce qui signifie qu'elle n'en a pratiquement pas). D'autre part, l'abbé avait remarqué au début de son discours du 2 thermidor que la Constitution n'est qu'une " partie » de cette machine.

La pensée constitutionnelle de l'an III contredit donc en plusieurs points les apparences de la loi fondamentale qu'elle produit : on ne peut pas tout prévoir dans les articles de l'acte constitutionnel. Dans cette perspective, on pourrait aussi dire que les constituants révolutionnaires essaient d'introduire dans la Constitution même ce qui en reste au-dehors, mais ce serait réduire l'importance de ce moment politique. Les événements historiques montrent que les membres de la Convention étaient allés audelà, plus encore dans les faits que dans la théorie. Il ne suffit pas de regarder dans les préceptes de la Constitution pour affirmer l'aveugle dévouement de l'assemblée à une organisation rigide et absolue par rapport au fonctionnement du système politique. Et, de même, on ne peut pas déduire du rejet du jury de Sieyès le fait que le courant de pensée explicitant l'impossibilité de contrôler intégralement le mouvement de la machine politique ne débouche sur rien de concret.

49 La «sentinelle politique " invoquée par Eschasseriaux aîné prend quand même corps quand l'Assemblée décrète que les deux tiers de ses membres feront obligatoirement partie du nouveau pouvoir législatif ${ }^{65}$. La discussion sur cette décision se déroule dans la partie du débat réservée aux " moyens de terminer la Révolution », c'est-à-dire, dans le langage révolutionnaire, surtout aux moyens d'assurer l'application de la Constitution. Il faut «conserver une grande majorité du corps législatif actuel, pour surveiller l'exécution de la Constitution à ses premiers pas »- déclare Delahaye ${ }^{66}$. Mais le décret des $2 / 3$ représente une vraie abdication préventive aux principes acceptés dans cette même constitution : les constituants brisent l'autonomie des pouvoirs, nient leur séparation, reconnaissent à la Convention une faculté qu'elle n'est pas légitimée à exercer, en " attentant à la souveraineté nationale qu'ils venaient de proclamer " ${ }^{67}$.

50 Les thermidoriens, en définitive, ont compris que l'histoire et la politique suivront un chemin différent de celui indiqué par leur créature constitutionnelle et en même temps la Constitution de l'an III, tout compte fait, ne se condamne point par elle-même. Le processus qui amène à sa "non-application " périodique s'amorce bien avant sa proclamation, tandis que l'expérience de son élaboration mettra beaucoup d'éléments essentiels à la disposition de la dernière phase révolutionnaire : l'an VIII, une phase trop souvent oubliée.

La raison et l'expérience : la pensée politique de l'an VIII

51 «La Francepeut s'arrêter dans la République; mais pour arriver à la monarchie mixte, il faut passer par le gouvernement militaire ", écrit Mme de Staël dans ses Réflexions sur la paix intérieur ${ }^{68}$. Les événements historiques lui donnent raison, mais, de cette façon, la fille de Jacques Necker inaugure une ligne interprétative qui réduit l'an VIII au coup d'État de Brumaire et à l'avènement du pouvoir militaire, en donnant du relief à la continuité pour ainsi dire en aval, vers le futur, et non en amont, vers le passé. Il existe, au contraire, des liaisons très importantes avec toute la théorie constitutionnelle 
révolutionnaire ${ }^{69}$, qui vont confluer dans un moment précis d'interprétation. Les grands principes de la Révolution sont révisés en vue d'une nouvelle organisation institutionnelle et les points d'appui tantôt idéologiques tantôt constitutionnels construits à partir de 1789 révèlent leur fragilité intrinsèque. "Souveraineté populaire ", "représentation", "division des pouvoirs", "fonction législative", "gouvernement ", «administration »: ce sont tous des éléments reconsidérés par la théorie brumairienne sous le jour de la distinction entre "principes» (ou «bases ») et « organisation » : «Il existe des principes constitutionnels, nous voulons tous maintenir ces principes; mais il n'existe plus d'organisation constitutionnelle ", affirme Lucien Bonaparte dans la séance clé du 19 brumaire $^{70}$, et par ces mots il juge en même temps la loi fondamentale de 1795 .

On peut avoir la preuve de ce procès de révision théorique, par exemple, dans le discours avec lequel Boulay de la Meurthe attaque le système institutionnel de thermidor et demande de parcourir «les branches principales de notre régime politique ; partout nous y trouverons des défauts essentiels [...]. Il faut donc s'élever à ces principes fondamentaux, ne plus voir la Constitution que dans eux, et nos obligations que dans leur maintien. Mais vouloir tenir à la partie réglementaire de la Constitution, ce serait de notre part un respect superstitieux et funeste $»^{71}$. Il faut donc réfléchir encore une fois sur la signification exacte de "constitution", puisque elle ne peut plus correspondre à "l'acte de partage des pouvoirs", c'est-à-dire, dans le nouveau langage politique, à «l'organisation». Les papiers manuscrits de Roederer le confirment $^{72}$.

53 En effet, à partir de ce moment - c'est encore Boulay qui le montre - l'argument de discussion deviendra le gouvernement ( « on n'est pas même d'accord sur le sens constitutionnel de ce mot») et l'«opposition constante» entre Exécutif et Législatif produite par les précédents actes constitutionnels.

Bien que la Constitution de l'an VIII soit élaborée en forme presque privée «chez Bonaparte " à partir d'un projet, ensuite dénaturé, de Sieyès (tout cela est assez bien connu et nous dit immédiatement qu'on ne peut guère avoir recours aux débats parlementaires pour reconstruire sa genèse), on ne doit pas croire qu'il n'existe pas un redoutable effort théorique derrière elle. Plusieurs projets furent conçus dans le but de concilier les positions de l'abbé et du général et, au moins, ceux de Daunou, Boulay de la Meurthe et Roederer atteignirent une forme organique ${ }^{73}$.

55 Il ne vaut donc pas la peine de trop s'arrêter sur le grand électeur (désormais connu) proposé par Sieyès et rejeté par Bonaparte : on y reviendra à propos de son éventuelle absorption "neutralisante » dans le Sénat-Conservateur. Il vaut mieux plutôt noter qu'au même moment et de façon assez particulière, deux tendances théoriques de la Révolution s'achèvent : le Premier Consul «chef suprême » dans le Triumvirat, en effet, réalise en même temps l'aspiration à un gouvernement fort qui ne soit pas simple " exécuteur » et à un gouvernement collégial. À en croire Boulay de la Meurthe, c'est Roederer qui propose la triade consulaire où une sorte de veto réciproque oblige au moins deux des trois consuls à agir d'un commun accord. Les manuscrits de Roederer, d'autre part, révèlent sa tentative de médiation entre le plan de Sieyès et celui de Boulay (dans lequel il apparaît un consul supérieur aux autres, désigné aussi avec le nom de « Président » $)^{74}$.

56 Mais le point essentiel de la théorie brumairienne du gouvernement est saisi avec clarté par Pierre Cabanis : "Qu'il y ait un président unique, ou un président et deux consuls, 
ou un grand électeur et deux élus responsables, n'importe ; il faut toujours que l'unité de pensée et d'action régularise la force centrale d'où partent tous les mouvemens [...] une qualité fondamentale dans le pouvoir exécutif, est l'unité de pensée et action $»^{75}$. Cette idée s'est donc finalement affirmée. Ce n'est pas un hasard si dans la nouvelle Constitution l'expression « gouvernement » l'emporte sur « exécutif " ${ }^{76}$.

Le pouvoir exécutif, en effet, toujours selon Roederer, «s'exerce au centre pour la direction et la procuration générale et sur toutes les parties de la République pour l'exécution ou l'administration. Le pouvoir exécutif est composé de volonté et de force ». L'administration est faite d'action et jamais de délibération. Au centre du système se développe la pensée du gouvernement et à la périphérie l'action. Et le fait que, juste à ce moment-là, le "pouvoir réglementaire " se manifeste en tous ses éléments essentiels représente dans ce sens un signal d'extrême importance ${ }^{77}$.

Une division de fonctions assure l'unité du pouvoir (et, avec lui, l'unité de pensée et d'action). Ça ne se passe pas différemment pour ce qui concerne le pouvoir législatif, divisé comme chacun le sait - sur le sillage d'une suggestion sieyèsienne de division du travail $^{78}$ - en proposition du gouvernement, discussion du Tribunat, votation du Corps législatif et éventuel jugement d'inconstitutionnalité du Sénat Conservateur. On pourrait en effet soutenir que la Constitution de l'an VIII essaie de sortir de la rigidité, plus encore que des pouvoirs, des fonctions. La nouvelle organisation institutionnelle se révèle de plus en plus comme le fruit d'un projet politique qui est avant tout pragmatique et ne prétend plus répondre aux raisons d'un ordre supérieur des choses. Cabanis affirme emblématiquement que la distinction entre Législatif, Exécutif et Judiciaire "n'est pas complètement dans la nature des choses $~^{79}$. La séparation des pouvoirs n'est plus « donnée », elle est "à faire ». Donc, il y a autant d'ordres que de Constitutions. Ce n'est pas un hasard si, en 1799, la conviction que la Constitution doit être jugée non pour ses qualités intrinsèques mais par le temps et les faits va s'imposer avec une vigueur tout à fait nouvelle.

La pensée constitutionnelle de l'an VIII va dans cette direction: une étape fondamentale est encore une fois représentée par la réflexion sur le pouvoir neutre et le pouvoir constituant.

Pour Roederer, le « vice capital » de la théorie constitutionnelle jusqu'à ce moment-là a été de reconnaître le pouvoir de réformer - et donc de conserver - la Constitution «à un corps constitué, à une partie de la Constitution $»^{80}$; un vice (comme on l'a déjà dit ) également remarqué par Daunou dans l'acte constitutionnel de 1795.

La question avait été considérée maintes fois par la pensée révolutionnaire. La Constitution de l'an VIII la résout en attribuant le contrôle en matière constitutionnelle au Sénat Conservateur, organe qui ne tombe pas dans l'impasse mise en évidence par Roederer. En effet, selon l'opinion de Sieyès, il «n'est rien dans l'ordre exécutif, rien dans le gouvernement, rien dans l'ordre législatif ${ }^{81}:$ c'est comme s'il était, bien que né de la Constitution, en dehors des pouvoirs constitués. Le Sénat n'est pas chargé, comme au contraire Sieyès l'espérait, de "maintenir la Constitution dans toute sa pureté », et l'article 21 établit seulement qu'« il maintient ou annule tous les actes qui lui sont déférés comme inconstitutionnels par le Tribunat ou par le gouvernement ».

Sieyès considérait le Sénat Conservateur comme l'héritier du jury conçu quatre ans auparavant, gardien de la Constitution et dépositaire du pouvoir de révision constitutionnelle ; Roederer et Daunou pensaient qu'il aurait dû juger des incompétences des pouvoirs supérieurs et, de cette façon, les conserver; Cabanis parle 
d'un vrai «Pouvoir surveillant» que les constituants de Brumaire auraient sérieusement pensé à instituer ${ }^{82}$. Il faut évidemment se souvenir du droit d'absorption imaginé par Sieyès, que Roederer décrit comme applicable à tout grand fonctionnaire et pas seulement au grand électeur : c'est-à-dire que le Sénat aurait pu «absorber » en lui-même l'homme politique devenant trop puissant et donc, en cette condition, dépassant ses limites constitutionnelles. À tout cela, en correspondance des fonctions "politiques", Roederer ajoute des «fonctions morales» qui auraient pu rendre le pouvoir neutre, " principe et organe de l'opinion publique ».

C'est peut-être la première fois que la pensée constituante de la Révolution s'arrête avec une telle décision sur la possibilé de donner corps à un pouvoir neutre, équilibrateur des autres pouvoirs et sentinelle de la Constitution. Mais, surtout, l'essai de rendre fluide la dynamique des institutions et d'intervenir dans les impasses de la pratique politique n'est jamais poursuivi avec une telle conscience du fait que, au fond, il est au-dehors du domaine de la loi fondamentale écrite : autrement dit, que faire face à ces problèmes signifie introduire dans la constitution ce qui en réalité ne relève pas de la Constitution elle-même. Et tout cela partage paradoxalement la scène avec l'idée claire et précise qu'il est impossible de planifier chaque circonstance politique à travers une acte législatif.

Le paradoxe, en effet, est seulement apparent. Les deux lignes parallèles, peut-être, finissent par se rencontrer. C'est probablement dans ce sens qu'il faut lire le refus final de réaliser les mécanismes projetés ainsi que la création d'une œuvre constitutionnelle dépourvue de moyens bien définis de modification. La Constitution de Brumaire, à la différence des autres actes constitutionnels de la Révolution, ne prévoit aucune procédure de révision, bien que, comme chacun le sait, la compétence générique du Sénat en matière constitutionnelle lui permettra de devenir protagoniste des changements suivants, effectués par Sénatus-consulte.

La recherche soigneuse de mécanismes d'adaptation et la décision de ne pas en réaliser produisent le même résultat: une Constitution prête aux changements. La machine politique a ses propres règles - laissait donc entendre Sieyès -, et l'an VIII comprend qu'on ne peut pas toutes les prévoir dans la loi écrite. Peut-être qu'alors s'en remettre à ces règles est, d'une certaine façon, une voie pour les prévoir, c'est-à-dire pour les prendre en considération dans un projet politique qui n'est pas limité à l'application de la Constitution. Il y a des "principes », bien sûr ; mais l'« organisation » autour d'eux peut bien changer.

théoriciens de 1799 - il ne faut pas sous-estimer ce fait - leur créature législative n'est pas nécessairement et intégralement bonne en elle-même : elle sera jugée exclusivement par les faits et par l'histoire. « C'est au temps seul qu'il appartient de nous faire connoître tout ce que la nouvelle Constitution renferme d'excellent et de défectueux ", écrit Cabanis ${ }^{83}$.

C'est la première fois qu'une Constitution n'est pas présentée a priori comme l'impérissable incarnation de toute aspiration politique et cela nous indique une perception désenchantée tantôt du caractère transitoire des ouvrages politiques, tantôt du pragmatisme nécessaire pour assurer n'importe quelle forme d'ordre.

Les événements qui suivent la proclamation de la Constitution de l'anVIII peuvent être jugés de plusieurs façons, toutes légitimes. Mais dans la pensée constitutionnelle qui accompagne sa genèse se trouve une grande leçon: une leçon, sous un certain angle, unique dans le panorama révolutionnaire et dont une partie probablement insuffisante 
a été acceptée dans les trop souvent « rigides » régimes constitutionnels des XIxe et xxe siècles.

\section{NOTES}

1.Édouard Laboulaye, « La question des deux chambres »dans Revue des deux mondes, mai-juin 1871, pp. 457-477, en particulier p. 462.

2.On pourrait en effet justement parler (comme le fait Roberto Martucci, «1789, la Repubblica dei foglianti. Dal re d'antico regime al primo funzionario di Stato » dans Storia Amministrazione Costituzione, n.1, 1993, pp. 61-106) de «legislativo-centrismo » pour souligner le déséquilibre politique vers le corps législatif plutôt que vers la loi. Pour des éclaircissements sur les rapports de cette expression avec celle de « légicentrisme », bien plus employée, $c f$. par exemple Pasquale pasquino, « Le républicanisme constitutionnel de E. Sieyès ", dans Droits, n. 17, 1993, pp. 67-77, et spécialement p. 69.

3.À ce propos je me permets de renvoyer à mon livre Governo e costituzione. $L a$ trasformazione del regime politico nell'età rivoluzionaria francese, Milano, Giuffrè, 1993. Voir aussi l'essai de Giuseppe G.Floridia, « Le pouvoir exécutif et l'administration dans les constitutions de la France révolutionnaire (1789-1799) », dans Roberto Martucci (sous la direction de), Constitution \& Révolution aux États-Unis d'Amérique et en Europe (1776-1815). Actes du Colloque international de Macerata (Italie), 15-17 avril 1993, organisé par le Laboratoire d'histoire constitutionnelle "Antoine Barnave » de la Faculté de Sciences Politiques de l'Université de Macerata, Macerata, Laboratorio di Storia Costituzionale, 1995, pp. 483-495.

4.Expression employée déjà par Antoine Jacques Boulay de la Meurthe dans son Essai sur les causes qui, en 1649, amenèrent en Angleterre l'établissement de la République, Paris, Baudouin, an VII, p. 14.

5.« Vi è un altro livello di analisi del dibattito, più fruttuoso e meno utilizzato di quello della prassi : il livello dei paradigmi teorici che orientano la discussione » (Mauro Barberis, Benjamin Constant. Rivoluzione, costituzione, progresso, Bologna, Il Mulino, 1988, p. 96). Ça ne signifie pas en absolu être obligé de considérer la Révolution comme un «bloc » unique, bien qu'en effet privilégier la continuité implique souvent la nécessité de travailler à un niveau d'abstraction élevée. François Furet avait raison de le souligner (Introduzione à François Furet [sous la direction de], L'eredità della rivoluzione francese, Roma-Bari, Laterza, 1989, pp. 3-22 et spécialement p. 20).

6.Les études de Lucien Jaume ( "L'État républicain selon de Gaulle », paru en deux parties dans Commentaire, $n^{\circ}$ 51, 1990, pp. 523-532 et n 52, 1990-1991, pp. 749-755 et " De Gaulle dans l'histoire française de la souveraineté », dans Institut Charles-deGaulle, De Gaulle en son siècle. Actes des Journées internationales tenues à l'Unesco - Paris, 19-24 novembre 1990, Paris, Plon, 1991-1992, vol. II, pp. 15-27) et ceux de Marcel morabito (Le chef de l'État en France, Paris, Montchrestien, 1995) sont très attentives à l'évolution historique et théorique du rôle du chef d'État en France. 
7.«Et le langage est souvent plus difficile à faire évoluer que les institutions » (Michel Verpeaux, « Lepouvoir réglementaire sous la Révolution », dans Droits, n. 17, 1993, pp. 113-124, en particulier p. 113). Sur les transformations du langage révolutionnaire $c f$. Jacques Guilhaumou, La langue politique et la Révolution française. De l'événement à la raison linguistique, Paris, Klincksieck, 1989.

8.Pierre-Louis Lacretelle, Sur le dix-huit brumaire. À Sieyès et à Bonaparte, Paris, les marchands de nouveautés, an VIII, p. 64.

9.Il s'agit d'un procès qui est évident quand Daunou affirme qu'il ne faut pas « faire envisager comme d'indifférents remplissages, des détails souvent précieux, des ressorts délicats, qui peuvent être, dans la machine politique, des leviers puissants quoique obscurs et pour ainsi dire inaperçus " : cf. Archives Parlementaires de 1787 à 1860. Recueil complet des débats législatifs et politiques des Chambres françaises, (Première Série, 1789 à 1799), Paris, Paul Dupont Éditeur, 1867-1905, (ensuite ici indiquées comme Arch. Parl.), vol. LXIII, p. 409 (26 avril 1793).

10.Une image, celle du « cercle constitutionnel », familière aux députés des assemblées révolutionnaires.Par exemple, pour Billaud-Varenne, les autorités constituées doivent être circonscrites « dans le cercle de leurs devoirs » (comme rapporte Olivier Jouanjan, «La suspension de la Constitution de 1793 », dans Droits, n 17, 1993, pp. 125-138, p. 125).

11.Sur l'organisation du pouvoir exécutif du roi dans la première Constitution révolutionnaire, on peut encore consulter Albert Larroquette, Le pouvoir exécutif dans la Constitution de 1791, Bordeaux, Imprimerie de l'Université, 1921.

12.Il s'agirait d'un discours à la Constituante tenu par Prugnon le 14 juillet 1791 (dans ce sens les remarques de Pasquale Pasquino, «La teoria costituzionale della monarchia di luglio ", dans Materiali per una storia della cultura giuridica, n. 2, 1988, pp. 377-400, en particulier p. 377).

13.Antoine Barnave, De la Révolution et de la Constitution (sous la direction de Patrice gueniffey), Grenoble, Presses Universitaires de Grenoble, 1988, p. 204: ce texte a été publié de façon posthume pour la première fois en 1843.

14.Jacques Necker, Du pouvoir exécutif dans les grands États, Paris, s.e., 1792, p. 15.

15.Dans le langage politique prérévolutionnaire, les mots « constitution » et « gouvernement » sont très proches l'un de l'autre: les deux se réfèrent à plusieurs égards à la structure essentielle du corps politique. Grâce aux concepts de " exécution » et « exécutif », au contraire, le gouvernement peut devenir « partie » de la Constitution. Pour les positions de Barnave cf. Arch. Parl., vol. XV, pp. 659-660 (22 mai 1790).

16.Jean-Joseph Mounier s'exprime de cette façon, en se référant à « un Gouvernement où un seul régit suivant la loi, où un seul est chargé de la faire exécuter » (Considérations sur les gouvernements et principalement sur celui qui convient à la France, Versailles, De l'Imprimerie de Ph. D. Pierres, 1789, p. 22).

17. Henry Bancal, Secondes réflexions sur l'institution du pouvoir exécutif, Paris, P. Beaufils, 1791, p. 2.

18.Barnave va jusqu'à dire que de cette question « dépend » la « conservation » de la Constitution même; pour cette intervention et pour celle de Gaultier de Biauzat, $c f$. Arch. Parl., vol. XV, p. 641 et 638 (21 mai 1790).

19.Sur la définition du concept de « constitution » à l'époque révolutionnaire, $c f$. Paolo Colombo, « Instaurazione, mantenimento e mutamento dell'ordine politico. La constitution nel lessico della rivoluzione francese », dans Filosofia Politica, n² 2, 1991, pp. 303-324. 
20.Arch. Parl., vol. XXIX, pp. 323-326 (10 août 1791). Sur l'intervention ici en question on ne peut tout de même pas oublier les remarques de Joseph Barthelemy (Le rôle du pouvoir exécutif dans les Républiques modernes, Paris, Giard \& Brère, 1906, pp. 432-440), qui maintient le discours centré sur la question monarchique en considérant Roederer incapable d'évaluer le rôle joué par le Roi comme titulaire de la dignité nationale. 21.Pour Rabaut Saint-Étienne (voir Jean-Paul Rabaut Saint-Étienne, Principes de toute Constitution, Versailles, Baudouin, s.d, reproduit dans Stéphane Rials [sous la direction de], La Déclaration des droits de l'homme et du citoyen, Paris, Hachette, 1988, pp. 675-684, en particulier l'art. IV « Du Gouvernement », p. 677). Pour l'opinion de Robespierre, voir Arch. Parl., vol. XXIX, pp. 326-327 (10 août 1791).

22.Arch. Parl., vol. XXIX, pp. 329-330 (10 août 1791). À propos de la distinction entre pouvoirs et fonctions dans la pensée des rédacteurs de la constitution de 1791, cf. toujours Léon Duguit, Traité de droit constitutionnel, Paris, Ancienne Librairie Fontemoing \& C., 1928, vol. II, pp. 531-532, qui nie l'existence d'une fonction exécutive dans le droit positif français.

23.L'épreuve inédite du discours de Sieyès est signalée par Pasquale Pasquino («Sieyès, Constant e il "governo dei modernii". Contributo alla storia del concetto di rappresentanza politica ", dans Filosofia Politica, n¹, 1987, pp. 77-98) aux Archives Nationales de Paris (284 AP 4 doss. 12). Pasquino a ensuite publié cette épreuve - avec d'autres inédits importants de l'Abbé - dans son Sieyès et l'invention de la Constitution en France, Paris, Éditions Odile Jacob, 1998. À ce propos, il faut signaler la récente sortie de Christine Fauré (sous la direction de), Des manuscrits de Sieyès 1773-1799, Paris, Honoré Champion Éditeur, 1999. Pour ce qui concerne l'intervention de Barnave, voir Arch. Parl., vol. XXIX, p. 331 (10 août 1791).

24.Benjamin Constant renvoie explicitement, à ce propos, aux Monarchiens et à Clermont-Tonnerre en particulier: $c f$. le passage, assez connu, de l'Esquisse de Constitution contenu dans les Réflexions sur les constitutions, la distribution des pouvoirs et les garanties, dans une monarchie constitutionnelle, Paris, H. Nicolle-Gide, 1814, pp. 175-176 ; mais aussi celui dans Fragments d'un ouvrage abandonné sur la possibilité d'une constitution républicaine dans un grand pays, inédit publié sous la direction de Henri Grange, Paris, Aubier, 1991, p. 398. Bien qu'on puisse avec raison formuler l'hypothèse que ce renvoi représente la couverture d'une opération théorique qui est seulement à Constant (voir, dans ce sens, Mauro Barberis, Benjamin Constant, cit., pp. 220-222, mais il ne faut pas oublier l'influence de l'abbé Sieyès, rappelée par Marcel Gauchet, Constant, in François Furet - Mona Ozouf [sous la direction de] Dictionnaire critique de la Révolution française, Paris, Flammarion, 1988, pp. 951-960). On ne peut pas nier, comme nous le verrons ensuite, que les racines de l'idée de pouvoir neutre sont probablement plutôt nombreuses et qu'on en trouve dans le milieu monarchien. Une intervention de Dominique La Rochefoucauld, qui décrit le rôle du roi par rapport à une Chambre unique comme « régulateur » de la « mécanique politique » [Arch. Parl., vol. VIII, pp. 548-549 (2 septembre 1789)], signale évidemment, en particulier, que cette idée est " semée » au début de la Révolution dans le « terrain » du veto.

25.Sur la recherche par Mirabeau d'un accord institutionnel entre Roi et Assemblée, voir Maria Sofia Corciulo, "Mirabeau et l'origine de la tactique parlementaire », dans Roberto Martucci (sous la direction de), Constitution \& Révolution, op. cit.., pp. 297-313. 26.Thouret affirme que « l'obligation de faire exécuter les lois, de mettre la force publique en activité [...] exige dans les grands États un chef qui soit le principe et le centre de tous les mouvements du corps politique » (Arch. Parl., VIII, 326, 1er août 1789). 
Anacharsis Cloots parle d'un « monarque » électif à l'américaine (Monarchie sans roi, dans Écrits révolutionnaires 1790-1794, Paris, Éditions Champ Libre, 1976 pp. 370-373, article qui parut dans les Annales patriotiques et littéraires du 27 septembre 1792). 27.Par exemple, Stanislas Clermont-Tonnerre (Analyse raisonnée de la Constitution française, dans CEuvres complètes de Stanislas de Clermont Tonnerre; Contenant ses opinions sur la Révolution française, et sur plusieurs gouvernements de l'Europe, Paris, Chez Letellier, an III, vol IV, pp. 298-300) trace le contour d'un Conseil exécutif comme « centre de gouvernement ». Brissot travaille aussi à l'idée d'un Conseil exécutif électif entre 1790 et 1792, et ses opinions présentent d'intéressantes concordances avec les modèles de Condorcet (de qui Brissot est très proche) : voir, à ce propos, Fernanda Mazzanti Pepe, Il mondo nuovo di Brissot. Libertà e istituzioni tra antico regime e rivoluzione, Torino, Giappichelli, 1996.

28. Un problème qui ne trouve pas de solution décisive dans la Révolution et qui a probablement un lien avec la question de la souveraineté (Paolo colombo, Governo e costituzione, op. cit., pp. 12 et 394); un problème qui est en même temps très actuel en termes de «théorie de la démocratie » (Marcel Gauchet, La Révolution des pouvoirs. La souveraineté, le peuple et la représentation 1789-1799, Paris, Gallimard, 1995, pp.15-16). 29.On sait que la Convention, non sans l'objectif tactique poursuivi par une partie de ses membres de différer l'approbation du nouveau texte constitutionnel, suspendit la discussion du plan de Condorcet et invita tous les citoyens intéressés à faire parvenir conseils, critiques et projets pour la future Constitution. Parmi ces matériaux, très importants pour comprendre l'attitude théorique du moment post-monarchique, on trouve aussi les écrits de Didier Thirion, La pierre angulaire de l'édifice constitutionnel, dans Arch. Parl., vol. LXVII, pp. 403-409 (24 juin 1793, Annexe) et de Pierre Charles François Dupont, Bases de la Constitution française, dans Arch. Parl., vol. LXVII, pp. 310-315 (24 juin 1793, Annexe). À propos de ce moment du débat constitutionnel de la Révolution, voir Michel Pertué, « Les projets constitutionnels de 1793 », dans Michel Vovelle (sous la direction de), Révolution et République. L'exception française, Paris, Kimé, 1994, pp. 174-199. L'effort de définition envers le « gouvernement représentatif » est clairement continué pendant l'an III et l'an VIII: $c f$. sur ce point Paolo Colombo Governo e costituzione, op. cit., en particulier p. 473.

30.Cf. Paul Bastid, Le gouvernement d'assemblée, Paris, Éditions Cujas, 1956, p. 186. Sur l'expérience du Conseil exécutif provisoire, voir aussi Joseph Barthelemy, Le rôle du pouvoir exécutif, op. cit.., p. 469 et Boris Mirkine-Guetzevitch, « Le gouvernement parlementaire sous la Convention ", dans Cahiers de la Révolution française, $\mathrm{n}^{\circ}$ 6, 1937, pp. 47-91, et en particulier pp. 66-67.

31.Cf. respectivement François Antoine Boissy d'Anglas, Projet de constitution pour la République française, dans Arch. Parl., vol. LXII, pp. 287- 315 (17 avril 1793, Annexe) et Pierre Claude François Daunou, Essai sur la Constitution, dans Arch. Parl., vol. LXII, pp. 350- 370 (17 avril 1793, Annexe).

32.Je dois encore renvoyer, ici, à mon Governo e costituzione, op. cit.., pp. 447-486. Cf. aussi, plus récemment, Marc Belissa, « Pouvoir exécutif, centralité législative : le débat sur l'organisation du ministère de la Guerre (janv.-fév. 1793) ", Annales historiques de la Révolution française, $\mathrm{n}^{\circ} 314,1998, \mathrm{pp} .699-718$.

33.Fondamental, à ce propos, le livre de Lucien Jaume, Le discours jacobin et la démocratie, Paris, Fayard, 1989, et en particulier, pour un premier schéma de « la remise en question de la représentation institutionnelle » (et de sa liaison avec l'idée qui existe une « partie saine » de l'Assemblée), pp. 78-80. 
34.Les Archives Parlementaires reproduisent le débat sur le gouvernement provisoire et révolutionnaire dans les versions de plusieurs journaux : vol. LXXX, pp. 363-369 (29-11-1793). La première phrase citée dans le texte est attribuée à Billaud-Varenne par l'Auditeur national, la deuxième par les Annales patriotiques et littéraires.

35.Pour une analyse du discours de la souveraineté du peuple (ainsi que des positions minimalistes et maximalistes à ce propos), voir Jacques Guilhaumou, « Un argument en révolution, la souverainté du peuple. L'expérimentation marseillaise », Annales historiques de la Révolution française, $n^{\circ}$ 4, 1994, pp. 695-714.

36. On parle évidemment, ici, de la réorganisation des Comités de salut public et de sûreté générale. Cf. L'ancien Moniteur, seule histoire authentique et inaltérée de la Révolution française depuis la réunion des États généraux jusqu'au Consulat, Paris, Henry Plon Imprimeur Éditeur, 1858-1863 (ensuite ici indiqué comme Mon.), vol. XXI, p. 585 (9 fructidor an II / 26 août 1794 ; séance du 7 fructidor an II ; pour mieux s'orienter dans le débat on donne ici les dates de publication du journal, autant que la date de séance). 37.Cf. Mon., vol. XXII, pp. 501-502 (26 brumaire an III / 16 novembre 1794 ; séance 24 brumaire an III). Barère et Tallien aussi saisissent immédiatement la balle au bond (pp. 503-506), mais l'Assemblée abordera effectivement le problème seulement au début de germinal, engageant la discussion qui amènera de manière significative à la formation de la Commission des Onze.

38.Id., vol. XXIV, p. 247 (1 floréal an III / 20 avril 1795; séance 29 germinal an III). 39.Thibaudeau est (selon Sergio Luzzato, L'autunno della rivoluzione. Lotta e cultura politica nella Francia del Termidoro,Torino, Einaudi, 1994, p. 298) « il deputato più lucido nell'intendere come una rifondazione della Repubblica dovesse passare prioritariamente attraverso una ristrutturazione del governo e della pubblica amministrazione "; voir ses interventions dans Mon., vol. XXIV, pp. 317-330 (10 floréal an III / 29 avril 1795 ; séance 7 floréal an III), pp. 322-323 (11 floréal an III / 30 avril 1795 ; séance 7 floréal an III), p. 354 (15 floréal an III / 4 mai 1795 ; séance 8 floréal an III), pp. 428-430 (24 floréal an III / 13 mai 1795; séance 20 floréal an III). Il faut considérer également, toujours d'Antoine Claire Thibaudeau, le Plan d'organisation d'un gouvernement intermédiaire, jusqu'à ce que la Constitution puisse être organisée, présenté à la Commission des Seize par la section qu'elle avoit chargée de cet objet, composée des Représentans du Peuple Thibaudeau, Romme et Merlino, Paris, Imprimerie nationale, an III et les volumes de Mémoires sur la Convention et le Directoire, Paris, Baudouin frères, 1824, en particulier vol. I, pp. 173-183. En plus, juste pour donner quelques points de référence dans un débat assez complexe: Cambacérès parle dans les séances du 29 germinal et du 11 floréal; Lesage du 10 floréal; Villetard du 11 floréal; Fréron du 21 floréal. $C f$. aussi les textes imprimés suivants: Jean-Denis lanjuinais, Opinion sur le gouvernement provisoire de la République, Paris, de l'Imprimerie nationale, an III ; Jean-Baptiste louvet, Opinion sur le gouvernement provisoire de la République, Paris, de l'Imprimerie nationale, an III ; Édmé Pierre Alexandre villetard, Opinion sur l'organisation provisoire du gouvernement de la République, Paris, de l'Imprimerie nationale, an III.

40.Voir les deux œuvres de 1797, Des causes de la Révolution et de ses résultats, publiée dans le Journal d'économie publique au mois d'avril 1797 (par Lezay) et Des effets de la Terreur (par Constant) paru la première fois comme introduction à la deuxième édition de Des réactions politiques, s.l., s.e., an V. Cette fracture dans le front libéral préfigure l'opposition de deux interprétations différentes de la Révolution, typiques surtout de l'historiographie du xixe siècle : $c f$., à ce propos, l'Introduzione de Mauro Barberis à la 
traduction italienne des écrits de Lezay et de Constant ici en question avec le titre Ordine e libertà, Torino, La Rosa, 1995, en particulier p. XXIV.

41.C'est encore Thibaudeau : Mon., vol. XXIV, respectivement p. 428 (24 floréal an III / 13 mai 1795 ; séance 20 floréal an III) et p. 318 (10 floréal an III / 28 mars 1795 ; séance 7 floréal an III).

42.Bertrand Barère (De la pensée du gouvernement, Genève, s.e., an V) met la pensée du gouvernement dans une " agence exécutive suprême » (le Directoire) et la simple action dans les ministres.

43.Cf., pour le discours de Cambacérès, Mon., vol. XXI, p. 474 (27 thermidor an II / 14 août 1794 ; séance 24 thermidor an II) et, pour la phrase de Jean-Jacques LenoirLaroche, l'article Quelques idées sur l'esprit que doit avoir le nouveau gouvernement, dans la rubrique « Mélanges », Mon., vol. XXVI, pp. 394-395 (20 brumaire an IV / 11 novembre 1795).

44.La lettre de Mme de Staël à Roederer est du 9 juin 1795 et se trouve dans la Correspondance générale de Mme de Staël, tome III, deuxième partie (Lettres d'une nouvelle républicaine. 17 mai 1795 - fin novembre 1796), Paris, chez Pauvert, 1972, pp. 14-17 ainsi que dans les Cuvres du Comte P.-L. Roederer, Paris, Firmin-Didot, 1853-1859, vol. VIII, p. 646. 45.Les papiers de la Commission des Onze sont conservés aux Archives Nationales de Paris. Je renvoie ici en particulier à la cote C. $232^{1} ; 183^{*}$ bis 14 , mais à propos de ces matériaux voir aussi l'analyse conduite sur les 884 envois parvenus à la Commission par Françoise Brunel (, dans Jean Nicolas (sous la direction de), Mouvements populaires et conscience sociale, XIVe-XIXe siècle, Paris, Maloine Éditeur, 1985, pp. 687-696).

46.Cf. Du Gouvernement, Paris, Impr. du Journal de Paris, 1795 : Roederer intervient dans les réunions des Onze (et, comme lui, Lamare: voir Patrice Gueniffey, « La révolution ambiguë de l'an III : la Convention, l'élection directe et le problème des candidatures ", dans Roger Dupuy - Marcel Morabito (sous la direction de), 1795 Pour une République sans Révolution, Rennes, Presses Universitaires de Rennes, 1996, pp. 49-78, en particulier p. 53).

47.Pour déclarer déchue la confiance, il faut une majorité des trois quarts des votes; la place de l'agent unique est alors prise par le président du Législatif et le conflit est jugé par un Conseil général des Corps administratifs municipaux et judiciaires. Si les deux tiers des votes du Conseil sont en faveur du décret du Législatif, les assemblées primaires sont convoquées pour la nomination d'un nouvel agent ou pour la réintégration de l'ancien. Voir toujours à ce propos les sources manuscrites de la Commission des Onze sous la cote AN. C. $232^{1} ; 183^{*}$ bis 14 ; en particulier, je renvoie ici au titre « Pouvoir Exécutif », inspiré aussi, probablement, aux différentes propositions parvenues à la Commission.

48.Le projet a pour titre Quelques vues sur l'organisation du pouvoir exécutif et est conservé sous la côte AN. C. $232^{1} ; 183^{*}$ bis 13 .

49.La proposition est marginale dans le débat: mais, aussi originale que complexe (cette deuxième chambre nomme et révoque aussi les ministres et participe à la formation des lois de manière à réunir deux pouvoirs et contredire le principe de leur séparation), nous indique à quel point se poussent les discussions. C'est le médecin Saléses qui envoya ce projet à la Commission des Onze (cf. AN. C. $232^{1} ; 183^{*}$ bis 12 ).

50.François Poultier, Du Pouvoir exécutif, Paris, Imprimerie Nationale, an III, en particulier p. 1; cet écrit est la publication d'une intervention à la Convention du 6 messidor an III. 
51.Cf. Mon., vol. XXV, pp. 100-101 (13 messidor an III/1er juillet 1795 ; séance 5 messidor an III).

52.Pour les points essentiels de ces débats, $c f$. Paolo Colombo, Governo e costituzione, op. cit., pp. 500-504.

53.Cf. Mon., vol. XXV, p. 519 (5 fructidor an III / 22 août 1795 ; séance 30 thermidor an III).

54.Sur cette phase du débat, voir Mon., vol. XXV, pp. 519-521 (5 fructidor an III / 22 août 1795 ; séance 30 thermidor an III) : «Cette proposition ne peut pas être appuyée » affirme André Dumont. « Elle est appuyée par Daunou et moi » - le fait taire avec décision Lanjuinais. Il ne faut pas oublier que Daunou et Lanjuinais peuvent être comptés parmi les défenseurs du pouvoir neutre.

55.Si on veut accepter les hypothèses de Basil Munteano (Les idées politiques de Mme de Staël et la Constitution de l'an III, Paris, les Belles Lettres, 1931, en particulier p. 56) et de Henry Grange (« Necker , Madame de Staël et la constitution de l'an III », dans l'ouvrage collectif Approches des Lumières. Mélanges offerts à Jean Fabre, Paris, Klincksieck, 1974, pp. 225-239).

56.Mon., vol. XXV, p. 488 (1er fructidor an III / 18 août 1795 ; séance 24 thermidor an III). Image, d'autre part, assez ambiguë: pierre philosophale comme recette qui change la matière brute en or ou comme chimère? Thibaudeau, dans ses Mémoires (op. cit., vol. II, p. 337) penche pour ce dernier sens.

57.Michel Troper, « Il concetto di costituzionalismo e la mederna teoria del diritto », dans Materiali per una storia della cultura giuridica, n. 1, 1988, pp. 61-81, et en particulier pp. 72-74 ; cet article est la traduction d'une communication présentée au Colloque Théorie et pratique du gouvernement constitutionnel (Paris, 17-19 septembre 1987). 58.Cf. les écrits de Benjamin Constant - partageant sur ce point les idées de Sieyès tirés de ses Souvenirs historiques (1830) et publiés sous le titre J'ai beaucoup connu Sieyès, dans Commentaire, n. 46, 1989, pp. 416-424, spécialement p. 421. De façon significative, pour Sieyès, le jury est assimilé à un « tribunal de cassation dans l'ordre constitutionnel » (Mon., cit., vol. XXV, 26 thermidor an III / 13 août 1795; séance 21 thermidor an III). On voit ici le croisement entre la route du pouvoir neutre et celle de la justice constitutionnelle (très bien analysé par Gustavo Zagrebelsky, La giustizia costituzionale, Bologna, Il Mulino, 1977, ici consulté dans la nouvelle édition de 1988, en particulier pp.11-18, où on trouve aussi une intéressante explication des motifs de l'échec du jury sieyèsien).

59. Ces traces sont assez nombreuses: les Onze vérifient en effet plusieurs propositions parvenues dans ce sens (voir AN. C. $232^{1} ; 183^{*}$ bis 14 ). Parmi les notes de la Commission on retrouve aussi, du reste, une longue et intéressante lettre anonyme en défense du jury de Sieyès où on préfigure un système institutionnel très proche de celui réalisé pendant l'an VIII. Au même auteur anonyme appartient une note adressée, déjà en période de discussion sur les lois organiques, au journal de Fréron L'orateur du peuple ( ${ }^{\circ}$ CXXVII, 23 prairal an III, pp. 278-279): dans ce cas on propose une " Assemblée conservatrice » qui fait appel au peuple si les actes du Législatif ou de l'Exécutif violent les droits. Thibaudeau aussi, d'autre part, se souvient dans ses Mémoires qu'on chercha à adapter le jury au projet de la Commission (vol. I, pp. 177-201) et que cette idée trouvait des partisans dans l'Assemblée. Il existe aussi de nombreux signes de la faveur de Roederer, de Lamare et de Daunou pour l'idée d'un pouvoir équilibrateur. Bien que très utile à faire, il ne suffit donc pas de conclure que l'idée d'un pouvoir neutre est une sorte de « lieu commun » de la culture politique thermidorienne (voir le livre de Sergio 
Luzzato, L'autunno della rivoluzione, op. cit., qui fournit des indications importantes sur les papiers de la Commission des Onze et sur les échos du pouvoir modérateur qu'on y retrouve: surtout pp.310-312). Les racines de l'idée de « compliquer les pouvoirs » en introduisant un équilibrateur sont à antidater [ $C f$. Michel pertue, Les projets constitutionnels, op. cit., p. 22, sur les précurseurs du jury de Sieyès dans les projets de Constitution parvenus à l'Assemblée constituante de 1793, et le Mémoire de DEA (année universitaire 1989-1990) de Bruno nicolle, Aux origines du contrôle de constitutionnalité: la jurie constitutionnaire de Sieyès (1795)]. Un personnage du niveau d'Adrien Duport parle déjà pendant l'été $1792 \mathrm{Du}$ Tribunat, ou du Pouvoir conservateur dans L'Indicateur ou Journal des causes et des effets (n. XXXIII, 21 juin 1792, pp. 125-126). D’autre part, on a déjà vu de quelle façon Benjamin Constant, en décrivant son pouvoir neutre, renvoie à la pensée des Monarchiens, et à Stanislas de Clermont-Tonnerre en particulier, c'est-à-dire à 1789. Toutefois, on trouve des traces de cette idée même au-dehors du terrain strictement monarchien. Stefano Mannoni [Une et indivisible. Storia dell'accentramento amministrativo in Francia. I. La formazione del sistema (1661-1815), Milano, Giuffrè, 1994] a consacré une partie de ses recherches aux inédits de Sieyès, en révélant que l'intuition de l'importance du pouvoir neutre naît en même temps que les autres parties $d u$ système théorique de l'abbé. Et à côté de la solution collégiale se développe aussi la solution monocratique, dessinée dans la désormais célèbre polémique de 1791 entre l'abbé et Thomas Paine (cf. spécialement pp. 289-290). De toute façon, sur l'évolution de l'idée d'un « tiers-pouvoir ", il faut maintenent absolument voir l'excellente reconstruction opérée par Marchel Gauchet dans son livre La Révolution des pouvoirs, op. cit. On se retrouve donc de plus en plus proches de découvrir que la nécessité d'un pouvoir équilibrateur des pouvoirs est dans la nature même de la pensée constitutionnelle et du projet constituant. Et une analyse dans ce sens de l'évolution théorique et institutionelle post-révolutionnaire - développée pas seulement jusqu'au « Hüter der Verfassung » de Carl Schmitt, mais aussi jusqu'aux régimes démocratiques de type présidentiel (à propos desquels $c f$. Nikolaus notter, Der Präsident des Republik als Pouvoir neutre nach der französischen Verfassung vom 4. Oktober 1958, Würzburg, Gugel, 1964) - produirait selon toute probabilité des éléments utiles à confirmer cette hypothèse.

60.Pour le discours de l'abbé, voir Mon., vol. XXV, pp. 291-296 (7 thermidor an III / 25 juillet 1795; séance 2 thermidor an III) et p. 297 ( 8 thermidor an III / 26 juillet 1795 ; séance 2 thermidor an III).

61. "Si vous voulez que la France soit libre, compliquez les pouvoirs suprêmes » - lit-on dans un projet envoyé à la Commission des Onze (bournat, Opinion du citoyen Bournat, Conservateur des Forêts Nationales du Département de Vaucluse, sur le projet de Constitution présenté par la Commission des Onze, s.l., s.e., s.d., p. 13).

62.Sur les idées de « technocratie » et de « gouvernement des gardiens » voir le numéro monographique de Filosofia Politica, n. 3, 1995, et en particulier l'article de Pier Paolo Portinaro, , pp. 389-406.

63.Mon., vol. XXV, p. 484 (1er fructidor an III / 18 août 1795 ; séance 24 thermidor an III).

64.Il s'agit du discours du 21 thermidor, où l'abbé, en déclarant tout d'abord qu'il intervient sur demande de la Commission, revient sur son projet de jury: Id., vol. XXV, p. 442 (26 thermidor an III / 13 août 1795).

65.Sur le « décret des deux tiers » cf. Charles Borgeaud, Établissement et révision des Constitutions en Amérique et en Europe, Le-Puy-en-Velay, Imp. Marchessou, 1893, p. 259 ; 
mais, beaucoup plus récemment, Marcel Morabito et Daniel Bourmand, Histoire constitutionnelle et politique de la France (1789-1958), Paris, Montchrestien, 19964, pp. 116-117 ; et surtout Mona Ozouf, « Les décrets des deux-tiers ou les leçons de l'Histoire ", dans Roger Dupuy - Marcel Morabito (sous la direction de), 1795 Pour une République sans Révolution, op. cit., pp. 193-209. Pour les positions d'Eschasseriaux, voir Mon., vol. XXV, p. 482 (30 thermidor an III / 17 août 1795; séance 24 thermidor an III) : "Vous avez besoin d'une sentinelle politique qui soit toujours là pour faire rentrer chaque pouvoir dans les limites que la loi lui a tracées, et qui en soit le constant régulateur»

66.Mon., vol. XXV, p. 534 (7 fructidor an III / 24 août 1795 ; séance 2 fructidor an III): et, en effet, Delahaye parle de «mise en activité provisoire » de la constitution.

67.Louis Villat le faisait noter déjà dans son ouvrage La Révolution et l'Empire (1789-1815). I. Les assemblées révolutionnaires (1789-1799), Paris, Presses Universitaires de France, 1936, p. 340. Toute la théorie du gouvernement représentatif, vue sous cet angle, n'a plus aucun sens: il suffit de rappeler qu'on arrive à discuter de substituer le tirage au sort au choix du peuple.

68.Anne Louise Germaine Staël-Holstein-Necker, Réflexions sur la paix intérieure (1795), dans Cuvres complètes de Mme la Baronne de Staël, Paris, chez Treuttel et Würtz, 1820, vol. II, pp. 95-172, p. 113.

69.Et pas seulement avec la pratique constitutionnelle du Directoire. Une connection, celle-ci, plus évidente et remarquée déjà - correctement, d'autre part - par Albert Soboul (La Révolution française, Paris, Éditions Sociales, 1962, ici consulté dans l'édition Gallimard, 1979, vol. II, p. 285).

70.Cf. Mon., vol. XXIX, pp. 895-896 (21 brumaire an VIII / 11 novembre 1799 ; séance 19 brumaire an VIII).

71.Le discours de Boulay est seulement résumé en forme indirecte par le Moniteur (vol. XXIX, p. 897, 21 brumaire an VIII / 11 novembre 1799 ; séance 19 brumaire an VIII) mais est au contraire présent dans l'anonyme Mémoires historiques sur le dix-huit Brumaire contenant les détails exacts et plus circonstanciés des deux Conseils..., Paris, s.e., an VIII, pp. 83-90. Il vaut la peine de considérer les mots de Boulay à propos de la séparation entre «bases » et « principes » de la Constitution: « les bases de la Constitution ou les principes généraux sont bons: ce sont les principes de tout gouvernement républicain, la souveraineté du peuple, l'unité de la république, l'égalité des droits, la liberté, le régime représentatif; mais l'organisation constitutionnelle, arrangée sur ces bases, est essentiellement vicieuse » (p. 89). La distinction est reprise aussi, par exemple, par Pierre-Louis Lacretelle: « Il falloit donc en sortir [de la Constitution], pour la conserver ; ou plutôt pour en conserver les bases, qui en sont le fond, il falloit en corriger les réglemens, qui ne sont que l'accessoire » (Sur le dix-huit brumaire, op. cit., p. 29). 72.En ouverture de ses notes sur « la rédaction de la nouvelle Constitution », Roederer écrit que " premièrement il ne faut pas l'appeler Constitution. $1^{\circ}$ parce que l'idée de stabilité n'est plus attachée à ce mot; mais bien l'idée contraire; $2^{\circ}$ parce qu'il n'a plus aucune solennité. $3^{\circ}$ parce [que] toute Constitution doit être hors de l'atteinte des pouvoirs constitués... » (pour les manuscrits de Roederer, conservés aux Archives Nationales de Paris, voir la cote 29 AP 79, et en ce cas particulier le folio 91). 73.Pour une première analyse des événements liés à la génèse de la Constitution de Brumaire on peut encore renvoyer au livre de Jean Bourdon, La Constitution de l'an VIII, Rodez, Carrère Éditeur, 1941. Il est utile aussi de lire dans quelques parties l'article de Raymond Guyot, dans Revue Historique, n. 111, 1912, pp. 1-31, mais, naturellement, il 
faut prendre le départ de la description opérée par Antoine Boulay de la Meurthe dans sa Théorie constitutionnelle de Sieyès, Paris, Chez Paul Renouard, 1836.

74.Pour les opinions de Boulay voir sa Théorie constitutionnelle de Sieyès, op. cit., pp. 48-49: " Roederer rédigea un projet, dans lequel le gouvernement était confié à trois consuls, dont l'un serait premier consul, et les deux autres simplement adjoints. Ces deux derniers ne pouvaient rien faire sans le premier, et le premier sans un des deux adjoints. Ce projet ne me plaisant pas, j'en présentai un autre, qui me paraissait s'éloigner moins de celui de Sieyès. Au grand électeur, tel qu'il l'avait conçu, je substituais, sous le titre de président, ou tel autre titre que l'on préférait, un chef suprême, assistant aux délibérations des deux consuls gouvernans, et statuant sur les conflits qui pourraient s'élever entre eux ». Ce projet - à en croire son auteur - est refusé par Napoléon. Les notes manuscrites de Roederer comparent deux schémas institutionnels: l'un (siglé $\mathrm{S}$.) résume les idées de Sieyès sur le grand électeur, l'autre (marqué par la lettre B.) dessine l'hypothèse de (Archives Nationales, 29 AP 79, folio 85). Pour les opinions de Roederer sur les institutions consulaires $c f$. folios 88 et 89 . Il faut enfin ajouter que même dans les articles projetés par Roederer paraît l'idée que le «Premier Consul de la République, préside les deux autres " (folio 94).

75.Cf. Pierre Jean Georges Cabanis, Quelques considérations sur l'organisation sociale en général, et particulièrement sur la nouvelle Constitution, Paris, de l'Imprimerie nationale, an VIII, p. 31 et pp. 43-44.

76.Prosper Poullet aussi le souligne déjà dans Les Institutions françaises de 1795 à 1814, Bruxelles, Librairie Albert Dewit, 1907, vol. II, p. 500.

77.À ce propos, $c f$. l'excellent livre de Michel Verpeaux, La naissance du pouvoir réglementaire 1789-1799, Paris, PUF, 1991. Pour les opinions de Roederer, voir Archives Nationales 29 AP 79, à la quatrième des pages annexes au folio 100.

78.Un " permanent souci sieyèsien ", celui de la division du travail, selon Stéphane rials ( «Sieyès ou la délibération sans la prudence. Éléments pour une interprétation de la philosophie de la Révolution et de l'esprit du légicentrisme ", dans Droits, n. 13, 1991, pp. 123-138 et spécialement p. 132) qui met en lumière les liaisons entre spécialisation des organes et distribution des fonctions dans la pensée sieyèsienne.

79.Pierre Jean Georges Cabanis, Quelques considérations sur l'organisation sociale en général, et particulièrement sur la nouvelle constitution, op. cit., p. 19.

80.Archives Nationales, 29 AP 79, folio 77.

81. Voir à ce propos Antoine Jacques Claude Joseph Boulay de la Meurthe, Théorie constitutionnelle de Sieyès, op. cit., p. 32.

82.Pour les opinions de Roederer et de Daunou cf. aussi Jean Bourdon, La Constitution de l'anVIII, op. cit., p. 61 et pour les observations de Cabanis sur le " pouvoir surveillant » cf. toujours Quelques considérations sur l'organisation sociale en général, et particulièrement sur la nouvelle constitution, op. cit., p. 32.

83.Pierre Jean Georges Cabanis, Quelques considérations sur l'organisation sociale en général, et particulièrement sur la nouvelle constitution, op. cit., p. 47. 


\section{RÉSUMÉS}

L'auteur montre que la question du pouvoir exécutif est centrale même dans l'évolution institutionnelle et le débat politique d'une révolution qui a été toujours considérée essentiellement comme «légicentriste». Une remarquable ligne de continuité, de ce point de vue, passe parmi les différentes phases révolutionnaires. La monarchie constitutionnelle ne peut pas être réduite au problème du sort de la royauté, ainsi que l'an VIII ne peut pas être réduit au coup d'État de Brumaire. De même, la période des Comités n'est pas seulement une phase de gouvernement d'exception et elle prépare l'emploi de l'idée de « gouvernement représentatif » et l'avènement $d u$ gouvernement collégial. Une analyse conduite par l'auteur sur les sources d'archives révèle aussi la pluralité de solutions élaborées par les Constituants thermidoriens. La nécessité de réduire la fracture que le début de la Révolution a produite entre "vouloir » et «agir» est donc bien claire dans le débat de 1789-1799 et se traduit dans la recherche de la "pensée du gouvernement» perdue. L'effort vers une séparation des pouvoirs moins rigide et des règles constitutionnelles plus élastiques y est bien visible.

The Issue of Executive Power in the Institutional Evolution and Political Debate of the Revolution.

The author shows that the issue of executive power is central to the evolving institutions and political debate of a revolution which has always been thought of as basically «legicentrist ». A remarkable continuity in this respect is to be found throughout all the revolutionary phases. Constitutional monarchy cannot be reduced to the problem of the fate of the royalty, just as Year VIII cannot be reduced to the coup of Brumaire. So too, the period of Committee rule was not only a phase of emergency government, but likewise paved the way for the idea of "representative government" and the advent of collegiate rule. An analysis of the archival records by the author also shows the multiple solutions developed by the Thermidorian constituents. The need to reduce the split produced in the early days of the Revolution between wishful thinking and getting things done, is thus very evident in the debate of 1789 to 1793 and is manifest in the search for the lost pensée du gouvernement. A striving for a less rigid separation of powers and more elastic constitutional rules is clearly discernible.

La questione del potere esecutivo nell'evoluzione istituzionale e nel dibattito politico rivoluzionario.

L'autore mostra che la questione del potere esecutivo è centrale anche all'interno dell'evoluzione istituzionale e del dibattito politico di una rivoluzione che, come quella francese, è stata sempre considerata essenzialmente "legicentrista". Una notevole linea di continuità, da questo punto di vista, attraversa le diverse fasi rivoluzionarie. La monarchia costituzionale non può essere "appiattita" sul problema del destino del re, così come l'anno VIII non può essere ridotto al colpo di stato del Brumaio. Allo stesso modo, il periodo dei Comitati non costituisce semplicemente una fase di governo d'eccezione e prepara l'impiego dell'idea di “"governo rappresentativo" e l'avvento del governo collegiale. Un'analisi condotta dall'autore sulle fonti d'archivio porta anche in luce la pluralità di soluzioni elaborate dai costituenti del Termidoro. La necessità di ricomporre la frattura che all'avvio della rivoluzione si è prodotta fra "volere" e "agire" risulta quindi ben chiara nel dibattito del decennio 1789-1799 e si traduce nella ricerca della perduta "pensée du gouvernement". Durante questa ricerca, lo sforzo diretto a realizzare una separazione dei poteri meno rigida e delle regole costituzionali più elastiche è chiaramente visibile. 
Demuestra el autor como es central el tema del podcr ejecutivo, incluso en la evolución institucional y el debate político de una revolución que fue siempre considerada como "logicentrista". Una continuidad revelante aparece dentro de las diferentes etapas revolucionarias. No se puede reducir la monarquía constitucional al problema del destino del rey, como no se puede reducir el año VIII al golpe de Estado de brumario. La época de los “ comités ”, no es solamente una epoca de gobierno de excepción: prepara el advenimiento del gobierno cologial. Un análisis de las fuentes de archivos revela también la pluralidad de las soluciones elaboradas por los constituantes de thermidor. Es muy clara la necesidad de reducir la fractura de los principios de la revolución entre "querer" y "actuar" en el debate de 1789-1799. Se traduce en la busca del "pensamiento de gobierno" perdido. Se puede ver un esfuerzo de separación menos rígida de los poderes y de las reglas constitucionales.

Die Frage der vollziehenden Gewalt in der institutionellen Entwicklung und der revolutionären politischen Debatten.

Der Autor zeigt, daß die Frage der vollziehenden Gewalt im Mittelpunkt der institutionellen Entwicklung und der politischen Debatten einer Revolution liegt, die immer als "legizentristisch" betrachtet wurde. Von diesem Standpunkt aus gesehen durchläuft eine bemerkonswerte Kontinuität die verschiedenen Stadien der Revolution. Die konstitutionelle Monarchie kann ebensowenig nur auf das Problem des Schicksals des Königtums wie das Jahr VIII auf den Staatsstreich von Brumaire reduziert werden. Auf dieselbe Weise ist die Zeit der "Ausschüsse" nicht nur das Stadium einer Ausnahmeregierung, sie kündigt schon die Verarbeitung der Idee einer parlamentarishen Regierungsform und das Zustandekommen der kollegialen Regierung an. Eine vom Autor unternommene Analyse der Archivquellen zeigt auch die Vielzahl der Lösungen, die nach Thermidor von den Abgeordneten der verfassunggebenden Versammlung vorgeschlagen wurden. Die Notwendigkeit, die Kluft zu überbrücken, die der Anfang der Revolution zwischen "wollen" und "handeln" aufgetan hat, tritt also ganz deutlich in den Debatten zwischen 1789 und 1799 hervor und zeigt sich vor allem in der Suche nach der verlorenen "pensée du gouvernement”. Das Bestrehen nach einer weniger straffen Trennung der Gewalten und geschmeidigeren Konstitutionsregeln ist offenbar.

\section{AUTEUR}

\section{PAOLO COLOMBO}

Università Cattolica del Sacro Cuore (Milan) 